\title{
Automatic Pavement Cracks Detection using Image Processing Techniques and Neural Network
}

\author{
Nawras Shatnawi \\ Department of Surveying and Geomatics Engineering, \\ Al-Balqa' Applied University, Al-Salt, Jordan
}

\begin{abstract}
Feature extraction methods and subsequent neural network performances were used in this research to impose proper assessment for distressed roads for a case study area in the North of Jordan. Object recognition method was used to extract roads cracks from airborne images acquired by drones. After images has been thresholded and the noise removed, digital image processing algorithms were applied to detect the presence of different crack types in the surface of pavement. In addition to that, the process was capable to automatically determine the length and the orientation of the cracks which were used as input for a neural network pattern recognition function designed for this purpose. Artificial Neural Network was used, tested and verified for cracks extraction. Different patterns and numbers of hidden layers were also investigated. The results revealed that using image processing techniques and neural network could detect pavement cracks with high accuracy.
\end{abstract}

Keywords-Artificial neural network (ANN); feature extraction; image processing; pavement crack

\section{INTRODUCTION}

Roads are one of the most important elements in the civilized communities. They play vital role in connecting villages, cities, districts, states, production lines, and even countries together. Due to frequent and high load of vehicles on roads, it is normal to have failures in the top surface (pavement) of the roads. Standard failures of pavement are called distresses which are divided into several types that affect the performance of the road; these distresses can be categorized as: Cracking, which is the major distress type in main roads, while in secondary roads, potholes, patches or rutting are often found $[1,2]$. Mainly, nineteen distresses do exist in flexible pavement. However, this study will focus on cracks evaluation and assessment. In order to maintain good performing roads, proper maintenance should be followed frequently based on accurate pavement inspections and surveys. This kind of inspections can be done by specialists who can monitor, acquire images, collect data and assess roads distresses. This traditional way of data collection has many drawbacks such as: labor intensive, time consuming, dangerous specially in highways and prone to subjectivity [3], [4] and [5]. Therefore; Automatic distresses data collection using digital imaging technology has been used by researchers since more than 50 years, this method reduces disturbance to the public traffic and road hazard to human inspectors during the survey [6], [7]. Different techniques were used for automatic cracks detection in roads pavements such as [15] and [16], Medina et al, [8] used Gabor filter to detect concrete tunnels cracks, the new used method was capable to detect cracks in any direction with an accuracy of $95.27 \%$. Zhang et al. [17] automatically detected cracks in subway tunnel with the application of highspeed complementary metal-oxide-semiconductor (CMOS) industrial cameras, using morphological image processing techniques and thresholding operations. Oliveira and Correia [9] applied a modified Otsu thresholding algorithm on digital images after dividing them into different zones and compared each pixel with the surrounding histogram. Fukuhara et al. [14] provided methods that could only judge whether a crack existed and the results were not good enough, while Zhang et al. [10] used neural networks for cracks detection in roads pavements. The used methodology of the current research has an accuracy of $82.5 \%$, which is much less than Zhang et al. [17] for example. However, it can be considered good considering the high sensitivity and precision which were $90 \%$ and $87 \%$ respectively. The previous works used different methodologies for automatic pavement cracks detection. Up to the author knowledge, the combination of bilateral filter, Canny algorithm, k-means clustering and a morphological filter with ANN for pavement cracks detection from airborne images is recent.

\section{WORK METHODOLOGY}

The followed methodology in this work consists of four main parts: Image enhancement and pre-processing, feature extraction, Neural network modeling and validation process as shown in Fig. 1.

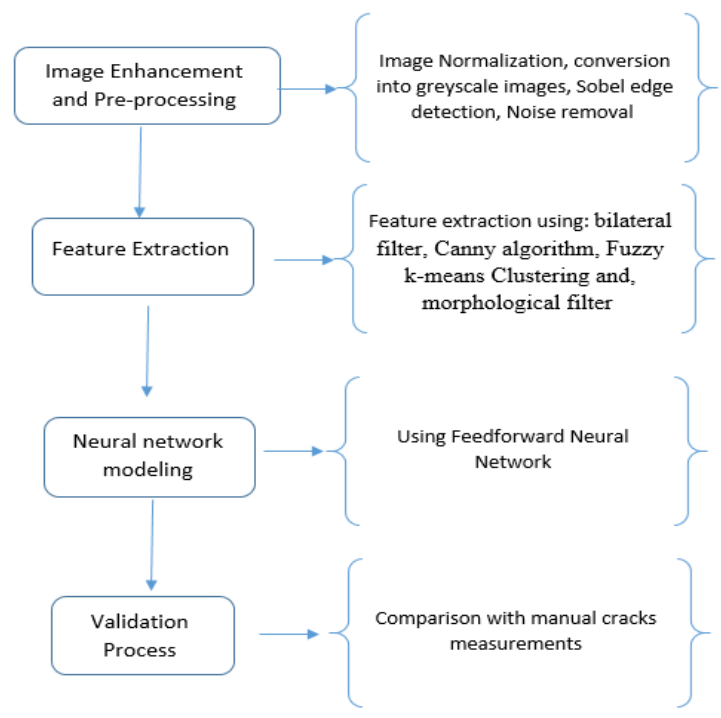

Fig. 1. Flowchart Showing the Methodology Followed in Conducting the Study. 

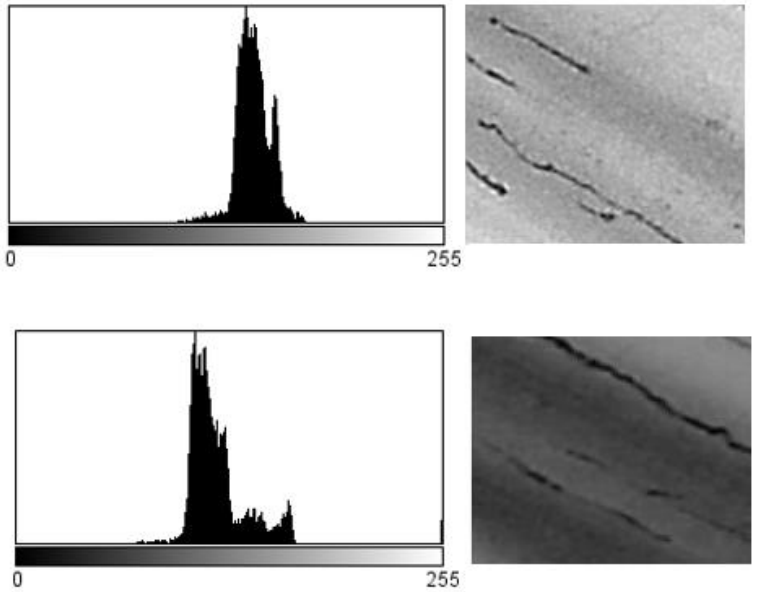

Fig. 2. Histogram of Road Pavement with Cracks.

In the first step; the acquired images by a drone were collected and preprocessed, the collected images were running through normalization function to average the intensity of the images, the images were then converted to grayscale, after that Sobel filter was used for edges detection followed by Noise removal filter. Thresholding was then applied to observe features. In fact, cracks can be identified by visual inspection which appears darker and different compared with road pavement pixels in the histogram as shown in Fig. 2. But unfortunately, the gray-level distribution of cracks is impossible to be separated from the gray-level distribution of the road pavement, therefore, image processing is required to support solving such problems.

Different extraction methods such as: bilateral filter, Canny algorithm, k-means clustering and a morphological filter were used, tested and compared. Among these methods, morphological filter 'closing' -which is simply a dilation followed by an erosion filter- this compound morphological operation showed the best results according to the minimum discrepancies between the actual and measured number of cracks and between the actual and measured length of cracks. The extracted features from the previous step were then used for pattern analysis and classification as input to the ANN module. In this research Feedforward Neural Network was used to simulate the extracted features. Finally, the extracted cracks values compared to manual cracks measurements on the road surface for model validation.

\section{DATABASE PREPARATION}

Sub-images were prepared from the captured aerial images, 800 image sets were used as source of data of cracked pavement and crack-free pavement as shown in Fig. 3.

Cropped images of the same crack were rotated to provide four more different directions for the ANN modeling as shown in Fig. 4.

In order to classify the images using ANN, data was divided as follows: Training set consists of $80 \%$ of data to build the model and determine the parameters (weights and biases), validation data set includes $10 \%$ of data to measure the performance of the network, and $10 \%$ of data is used to increase the robustness of the model in the test phase. The structure of the used ANN is shown in Fig. 5, in which Variable Learning Rate Backpropagation learning function is used to train the network.

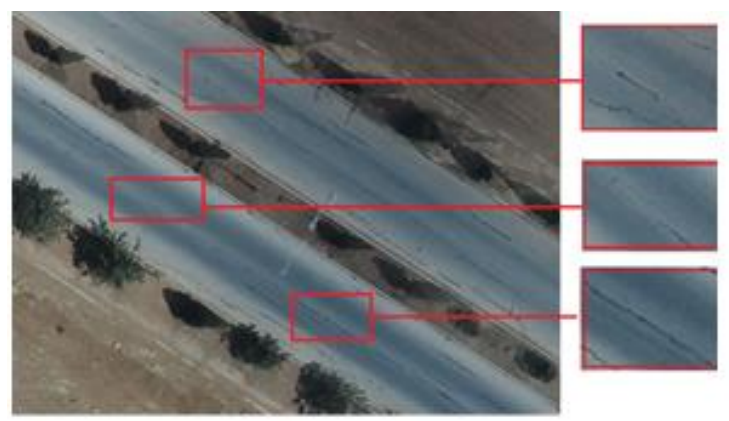

Fig. 3. Database Sampling from Images Subsets.
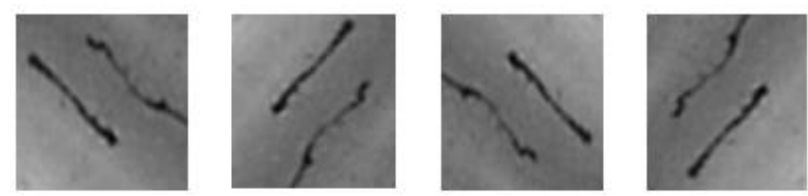

Fig. 4. Cropped Image of the Same Crack Rotated to Provide Four Different Directions.

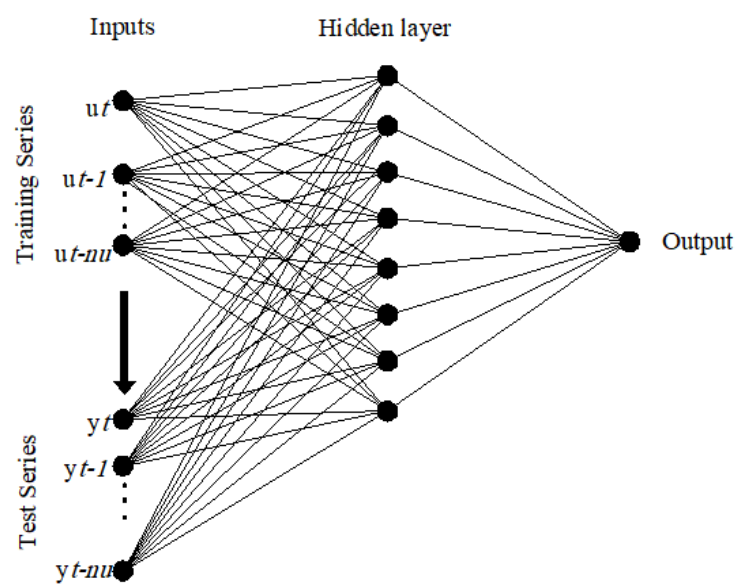

Fig. 5. Structure of the used ANN Model for Cracks Detection Model.

TABLE I. OPTIMAL NUMBER OF HIDDEN NEURONS IN THE HIDDEN LAYER

\begin{tabular}{|l|l|}
\hline $\begin{array}{l}\text { NUMBER OF } \\
\text { NEURONS }\end{array}$ & MEAN SQUARED ERROR (MSE) \\
\hline 1 & 0.0324 \\
\hline 2 & 0.0281 \\
\hline 3 & 0.0216 \\
\hline 4 & 0.0201 \\
\hline 5 & 0.0171 \\
\hline 6 & 0.0113 \\
\hline 7 & 0.0101 \\
\hline 8 & 0.0067 \\
\hline 9 & 0.0078 \\
\hline 10 & 0.0092 \\
\hline
\end{tabular}




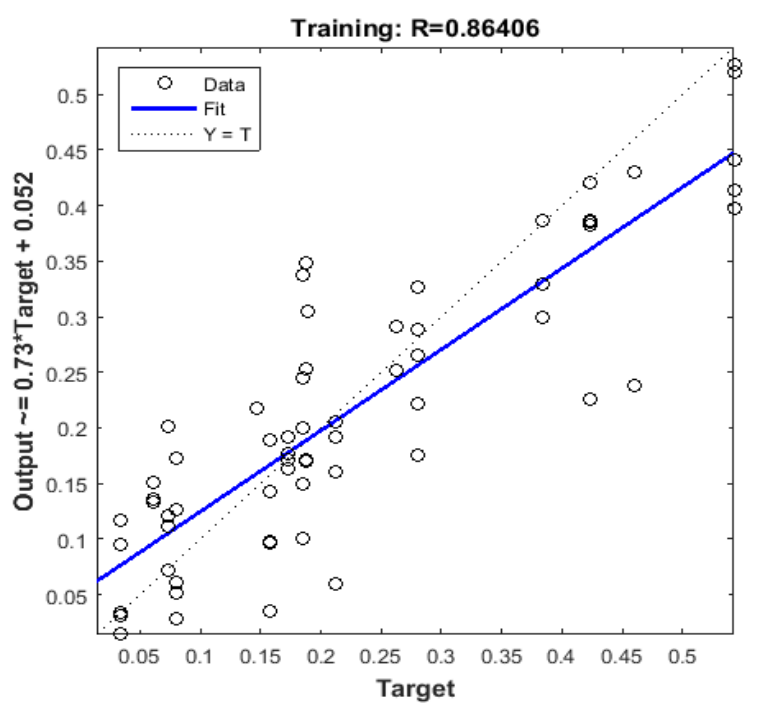

Fig. 6. Performance of the used ANN Model.

One hidden layer of 8 neurons is used in this network. The optimal number of neurons was identified after performing many trials based on the minimum value of mean squared error (MSE) as shown in Table 1.

Fig. 6 shows the performance of the used model, it presents the correlation between data points and curve fitting plots for training. It can be clearly noticed from this figure that the regression is approximately 0.864 which can be considered high enough to reflect a good relationship between the targets and the outputs.

\section{RESULTS AND DISCUSSION}

Four measures in this analysis were carried out; the true positives which are the samples that are correctly classified as cracks, true negatives are samples that are correctly classified as non-cracks, where, false positives are the samples that are not cracks but wrongly classified as cracks by the network and false negatives are crack samples but wrongly classified as non-cracks by the network [11]. Table 2 summarizes these four measures for a subset of 80 images.

Precision $=\frac{\text { True Positive }}{\text { True positive }+ \text { False positive }}$

TABLE II. TEST PERFORMANCE PARAMETERS

\begin{tabular}{|c|c|c|c|}
\hline $\begin{array}{c}\text { Test } \\
\text { Results }\end{array}$ & Present & Absent & Total \\
\hline Positive & True Positive $_{(54)}(8)^{\text {False Positive }}$ & 62 \\
\hline Negative & $\begin{array}{c}\text { False } \\
\text { Negative (6) }\end{array}$ & $(12)^{\text {True Negative }}$ & 18 \\
\hline Total & 60 & 20 & 80 \\
\hline
\end{tabular}

In order to determine how well this cracks detection method performs; sensitivity, specificity, precision and accuracy were computed for the same subset of images. The sensitivity (recall) of a test quantifies its ability to correctly identify subjects with the condition [12], in other words, it is the percent of crack samples that are identified by the network out of the total number of cracks in the dataset [11], given by:

Sensitivity $=\frac{\text { True positive }}{\text { True positive }+ \text { False negative }}$

Specificity is the ratio of true negatives divided by summation of true negative and false positives. It represents the probability of a test without giving false-positive results, given by [13]:

Specificity $=\frac{\text { True negative }}{\text { True negative }+ \text { False positive }}$

A precision is the percent of predicted cracks that were actually cracks, given by:

Accuracy is another factor for performance measurement, it represents the proportion of true results (both true positive and true negative) in the selected population.

Accuracy $=\frac{\text { True Positive }+ \text { True negative }}{\text { Total }}$

A summary of the mentioned factors for the tested subset is shown in table 3 .

TABLE III. SENSITIVITY, SPECIFICITY, ACCURACY AND PRECISION VALUES OF THE PERFORMED MODELING

\begin{tabular}{|l|l|}
\hline PARAMETER & RESULT \% \\
\hline Sensitivity & 90 \\
\hline Specificity & 60 \\
\hline Accuracy & 82.5 \\
\hline Precision & 87 \\
\hline
\end{tabular}

\section{CONCLUSIONS}

Extraction of cracks in roads pavement from airborne images was automatically detected using artificial neural network. The used ANN model showed a suitability for pattern recognition for roads cracks based on the high values of performance parameters. The proposed method opens the door for numerous applications of ANN on distresses classifications and measurements. It has been shown that the performance of the used feedforward neural network increased up to a certain number of neurons in the hidden layer. In contrast, some samples were predicted wrongly as false negative and false positive which represents fake cracks in the realty, such problems might be related to image noise and image interference like: shadows, lane line and trees branches. It is recommended that future research should cover the issue of image acquiring and accuracy assessment of the system and its effect on the extracted cracks.

It is expected that the proposed technology, setup configuration and procedures of automatically extracting 
pavements cracks will open the door for other applications in the domain of transportation engineering, road construction, and other domains. Moreover, we are looking forward to having hand-held cell phones applications of this technology on the smart phones themselves.

\section{CONFLICT OF INTEREST DisClOSURE}

The author(s) declare(s) that there is no conflict of interest regarding the publication of this paper.

\section{REFERENCES}

[1] H. Oliveira, "Crack detection and characterization in flexible road pavements using digital image processing" Thesis in electrical and computer engineering, Universidade Técnica De Lisboa, Instituto Superior Técnico. 2013

[2] T. Al-Suleiman, M. Kheder, and H. Al-Masaeid, "Development of Pavement Performance Models for Rural Roads," Journal of Road and Transport Research, Australian Road Research Board, Vol. 1, No. 4. 1992.

[3] D. Meignen, M. Bernadet, and H. Briand, "One Application of Neural Networks for Detection of Defects Using Video Data Bases: Identification of Road Distresses," IEEE 8th International Workshop on Database and Expert Systems Applications - DEXA, 1-2 September 1997, Toulose, France, pp. 459-464.

[4] Cheng, H. and Miyojim, M. "Automatic Pavement Distress Detection System," Information Sciences, vol. 108, no. 1-4, July, pp. 219-240. 1998

[5] P. Subirats, O. Fabre, J. Dumoulin, V. Legeay, and D. Barda, “A Combined Wavelet-based Image Processing Method for Emenrgent Crack Detection on Pavement Surface Images," 12th European Signal Processing Conference - EUSIPCO, 6-10 September 2004, Vienna, Austria, pp. 257-260.

[6] Wang, K. "Design and Implementation of Automated System for Pavement Surface Distress Survey," Journal of Infrastructure System, 6 (1), 24-32. 2000
[7] J. Zhou, P. Huang, and F. Chiang, "Wavelet-Based Pavement Distress classification," Journal of the Transportation Research Board, no. 1940, pp. 89-98, 2005.

[8] R. Medina, R., Llamas, J. Bermejo, E. Zalama, and M. Segarra, "Crack Detection in Concrete Tunnels Using a Gabor Filter Invariant to Rotation," Sensors, 17 (1), pp1670,2017.

[9] H. Oliveira, P.L. Correia, "Automatic road crack segmentation using entropy and image dynamic thresholding," In Proceedings of the 17th European Signal Processing Conference (EUSIPCO'2009), Glasgow, UK, pp. 622-626.

[10] L. Zhang, F. Yang, Y.D. Zhang, Y.J. Zhu, "Road crack detection using deep convolutional neural network," In Proceedings of the IEEE International Conference on Image Processing (ICIP), Phoenix, AZ, USA, 25-28 September 2016; pp. 3708-3712.

[11] R. Fuentes, L. Pauly, and D. Hogg, "Deeper Networks for Pavement Crack Detection," 34th International Symposium on Automation and Robotics in Construction (ISARC 2017).

[12] H. Wong, and G. Lim, "Measures of Diagnostic Accuracy: Sensitivity, Specificity, PPV and NPV," Proceedings of Singapore Healthcare, 2011, 20 (4), 316-318.

[13] H. Brenner, O. Gefeller, "Variation of sensitivity, specificity, likelihood ratios and predictive values with disease prevalence," Statistics in Medicine, 16: 981-991. 1997.

[14] T. Fukuhara, K. Terada, M. Nagao, A. Kasahara, and S. Ichihashi, "Automatic Pavement-Distress-Survey System," J. Transportation Engineering, ASCE 116 (3), 1991, pp 280-286,

[15] L. Sjogren, (2002) "State of the art: Automated Crack Measurement of Road Surface," research project report ,Swedish National Road and Transportation Research Institute (VTI). 2002.

[16] B. Xu, and Y. Huang, "Development of an automatic pavement surface distress inspection system," research project report, Center for Transportation Research at The University of Texas at Austin. 2005.

[17] W. Zhang, Z. Zhang, D. Qi, and Y. Liu, "Automatic crack detection and classification method for subway tunnel safety monitoring," Sensors, vol. 14, no. 10, pp. 19307-19328. 2014. 